\title{
Direct Observations of Energy Transfer and Quenching Dynamics between Alq3 and C545T in Thin Films with Different Doping Concentrations
}

\author{
Jiun-Haw Lee*, Chih-Chung Teng, Jian-Hong Lin, Tian-Chiun Lin and C. C. Yang \\ Graduate Institute of Electro-Optical Engineering and Department of Electrical Engineering, \\ National Taiwan University, No. 1, Sec. 4, Roosevelt Road, Taipei, Taiwan, R.O.C.
}

\begin{abstract}
In this paper, we conducted photoluminescence (PL) and time-resolved photoluminescence (TRPL) measurements for the organic films that was composed of tris(8-hydroxyquinoline) aluminum ( $\left.\mathrm{Alq}_{3}\right)$ as the host and 10(2-benzothiazolyl)-1, 1, 7, 7-tetramethyl-2, 3, 6, 7-tetrahydro- $1 \mathrm{H}, 5 \mathrm{H}, 11 \mathrm{H},[\mathrm{l}]$ benzo-pyrano [6,7,8-ij] quinolizin-11-one (C545T) as the green dopant with different concentration. Typical quench behavior was observed by typical PL measurements when doping concentration exceeds $2 \%$ and the carrier lifetime decreased monotonically with increasing doping concentration in TRPL measurements. Time constant for energy transfer from host to dopant decreased with increasing dopant concentration and saturated above $2 \%$ dopant concentration. An anomalous blue shift at the initial probe time-interval was observed when dopant concentration was over $4 \%$. It indicated a fast event energy absorption and/or relaxation process which had a time constant less than two picosecond. Two physical mechanisms with different time constant was observed those accounted for the concentration quench behaviors in the Alq3/C545T system.
\end{abstract}

Keywords: OLED, carrier dynamics.

\section{INTRODUCTION}

Organic light-emitting device (OLED) has attracted much attention because it exhibits the advantages of low-power consumption, high brightness, high contrast, and low cost [1],[2]. In such a device, doping high internal quantum efficiency (IQE) material inside the charge transport material to form an emitting layer (EML) can effectively increase the spectral region of the emission, increase device lifetime, and reduce unwanted self-absorption [3],[4],[5]. However, when doping concentration increased, the excited dopants will form ecimers or aggregates that were generally called concentration quenching [6],[7]. Once the excitation energy was trapped on an aggregate, the aggregate might come to the ground state either by the emission whose wavelength is different from that of the excitons or by the nonradiative relaxation.

In such a host-dopant material system, Förster energy transfer dominates the process [8]. This physical mechanism involves long-rang dipole-dipole coupling and results in the transfer of the excited-state energy from a host donor to a guest acceptor. Singlet exciton transfer from the host by Förster transfer and the energy level of singlet in host has to lie above the corresponding level in guest. Moreover, efficient transfer requires an overlap of emission in the host and absorption in the guest.

With increasing concentration of a guest acceptor, efficient energy transfer would be achieved. However, it also results in the concentration quench effect. Typically, energy transfer and concentration quench are two of the most important topics in OLED carrier dynamics [9],[10]. They played crucial roles in OLED device design for achieving

*jhlee@cc.ee.ntu.edu.tw; phone 8862 2363-5251 ext. 540; fax $88622367-7467$ 
high efficiency and long lifetime. In this paper, we analyze the energy transfer process in the thin film. The thin films we used were composed of tris(8-hydroxyquinoline) aluminum $\left(\mathrm{Alq}_{3}\right)$ as the host material and 10- (2-benzothiazolyl)-1, 1, 7, 7-tetramethyl-2, 3, 6, 7-tetramethyl $H, 5 H, 11 H,[l]$ benzo-pyano [6,7,8-ij] quinolizin-11-one (C545T) as the green guest material with different concentrations. We observed the energy transfer time decreased from 1.4 ns to about 400 ps with increasing dopant concentration from $1 \%$ to $2 \%$. However, the energy transfer time is slightly decreased to 300 ps when the dopant concentration is further increased to $5 \%$. Quench effect is clearly seen in the typical PL study when dopant concentration exceeds $2 \%$. In TRPL study, carrier lifetime decreased monotonically with increasing dopant concentration due to the increase of non-radiative recombination rate. We also observed that spectrum at the beginning of decays shows a red shift followed by a blue shift in increasing dopant concentration. The red shift means the faster energy transfer from the host to dopant with higher doping concentration and the blue shift shows the excimer absorption or non-radiative recombination at the red edge of the spectrum. Time constant of such a process is shorter than 2 ps that is the limit of our experiment setup. The experimental results and the related discussions are represented in section 2. Conclusions are drawn in section 3.

\section{EXPERIMENT RESULTS AND DISCUSSIONS}

In our experiments, the thin organic films were deposited on the glass substrate by a ULVAC multi-chamber vacuum evaporator with the thickness of $50 \mathrm{~nm}$. Prior to evaporation, the surface of the glass was cleaned by $\mathrm{O}_{2}$ plasma to remove the impurities. Five samples were fabricated with different dopant concentration, e.g., $0 \%, 1 \%, 2 \%, 4 \%$ and $5 \%$, which were labeled as A, B, C, D and E, respectively. The photoluminescence (PL) measurement was carried out at room temperature with a $325-\mathrm{nm}$ line $\mathrm{He}-\mathrm{Cd}$ laser to obtain the relative quantum efficiency, full width at half maximum (FWHM) and the peak wavelength of different samples. The time-resolved photoluminescence was performed by using a Hamamatsu streak camera (Hamamatsu Photonics KK, Japan) in conjunction with a 25-cm monochromator using a 600 lines $/ \mathrm{mm}$ grating. The excitation pump beam of $\lambda_{\mathrm{exc}}=400 \mathrm{~nm}$ was from the second-harmonic generation of a mode-locked Ti:sapphire laser with a pulse width of 100 femtosecond and a repetition period of $13.16 \mathrm{~ns}$.

Photoluminescence (PL) spectrum of different samples is shown in Fig. 1. With increasing doping concentration, PL intensity increases and spectrum peak red-shifts since energy transfer from host to dopant is more efficient. Maximum PL intensity is achieved at $2 \%$ doping concentration of sample $\mathrm{C}$. When doping concentration is over $2 \%, \mathrm{PL}$ intensity decreases and shows a clear concentration quenching effect. The result is consistent with other reports [11]. Fig. 2 shows the integrated intensity, spectrum peak and full width at half maximum (FWHM) variations with different dopant concentration. Integrated intensity has a maximum value at $2 \%$ dopant concentration. Spectrum peak is shift from 521.5 to $527 \mathrm{~nm}$ with increasing dopant concentration. These two wavelength values are used as probe wavelength in the following measurements. FWHM is decreased from $80 \mathrm{~nm}$ to $40 \mathrm{~nm}$ when C545T doped into the Alq3 and is almost kept at the same value with different doping concentration.

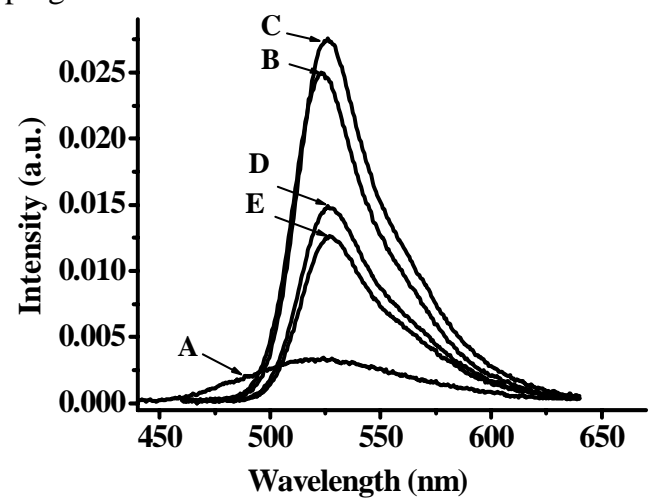

Fig. 1. PL spectrum of different dopant concentrations. 


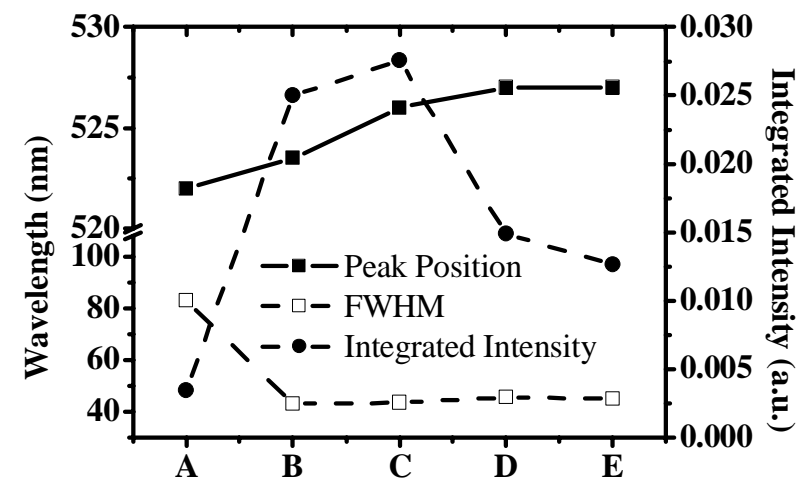

Fig. 2 Characteristics in PL spectrum of different dopant concentrations.

Fig. 3 shows the time constant of the time dependent intensity decay curve with different dopant concentration at the wavelength of 521.5 and $527 \mathrm{~nm}$, which corresponds to Alq3 and C545T spectrum peaks, respectively. Time constant for the dopant probe $(527 \mathrm{~nm})$ is always larger than that for the host probe $(521.5 \mathrm{~nm})$ except in the undoped condition (sample A). That is a energy transfer behavior from host to dopant material. When dopant concentration increases, time constant decreases that may be due to higher energy transfer probability. Another possible mechanism is the absorption that comes from the concentration quench. Typically, the model of quasi-planar molecular packing induced dye-dye interaction is used for explaining such a phenomenon. It is difficult to separate the energy transfer and the optical absorption effect with such kind of analysis. Besides, since the spectral overlap between Alq3 and C545T is large, there exists dopant (host) contribution in the host (dopant) probe wavelength of $521.5 \mathrm{~nm}(527 \mathrm{~nm})$. That also increases the difficulty in analyzing the experimental data.

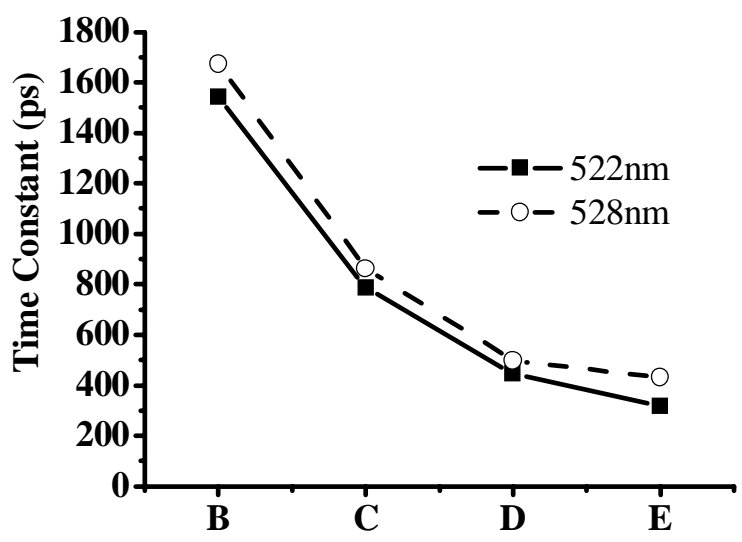

Fig. 3 Time constant with different doping concentration. 


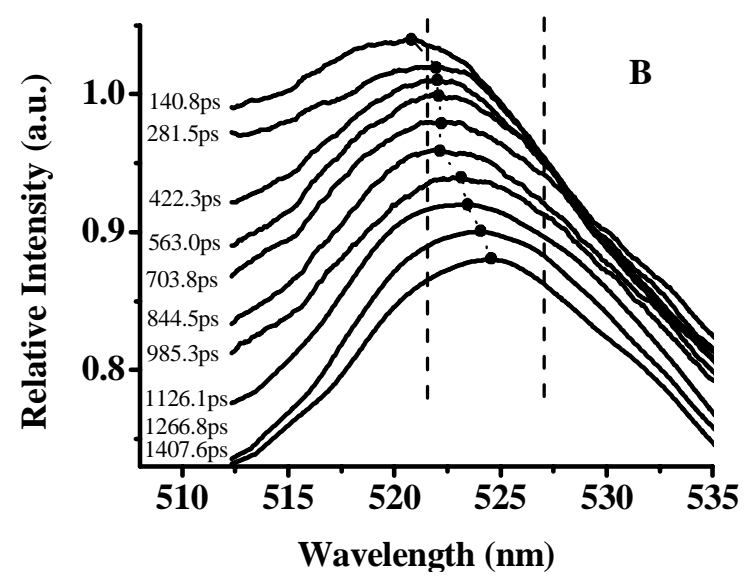

Fig. 4 Spectrum evolution of sample B.

Fig. 4 shows the spectrum of sample B taken at different time interval. We could observe that the peak position had a red shift with increasing time that was a typical energy transfer behavior from 140.8 ps to $1407.6 \mathrm{ps}$. When monitoring the relative intensities of the host and dopant probe wavelengths with different time intervals, we can see a clear cross-over at $1407 \mathrm{ps}$ as shown in Fig. 5. We define the energy transfer time at which the intensities at $521.5 \mathrm{~nm}$ and 527 $\mathrm{nm}$ are equal. The transfer time is plotted for different samples in Fig. 6. With increasing dopant concentration, the energy transfer time decreases rapidly and then saturates at high concentrations. Such a behavior is attributed to the finite length of the Förster energy transfer process in real space. The Förster energy transfer depends on the ratio of host-guest concentration and was a long-range energy transfer. The long transfer time is occurred by weak interaction of low concentration ratio. When doping concentration increases, the Förster energy transfer between host and guest materials raises and enables a large decrease in transfer times. However, the monotonically decreasing carrier lifetime with increasing dopant concentration as shown in Fig. 3 shows that optical absorption do play a certain role at higher dopant concentration.

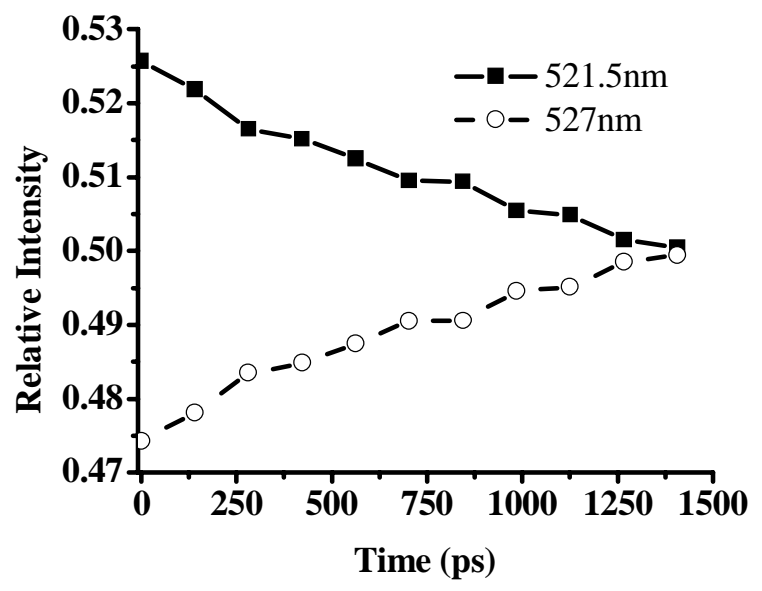

Fig. 5 Relative intensity at pump and probe wavelength of sample B. 


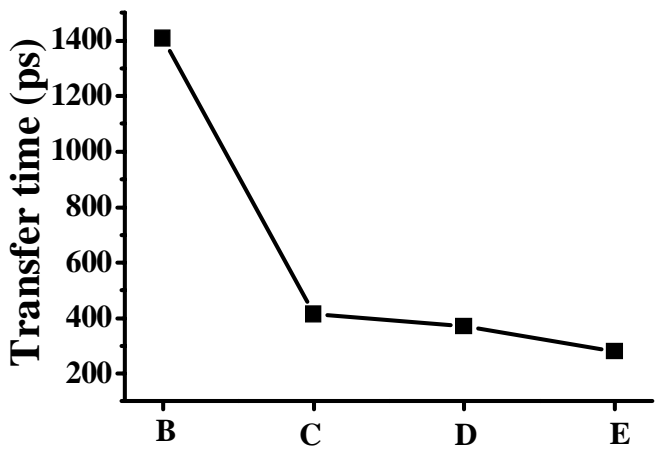

Fig. 6 Transfer time of different samples.

Fig. 7 shows the spectrum at the beginning of decay. A red shift followed by a blue shift in increasing dopant concentration is observed. Since higher concentration results in a higher energy transfer probability, the red shift behavior should be observed. The blue shift at high concentrations might result from aggregate absorption or excimer energy relaxation at the red edge. Although the time constant of such a mechanism cannot be calibrated in the current experiment, another mechanism does exist for explaining the blue-shift behavior.

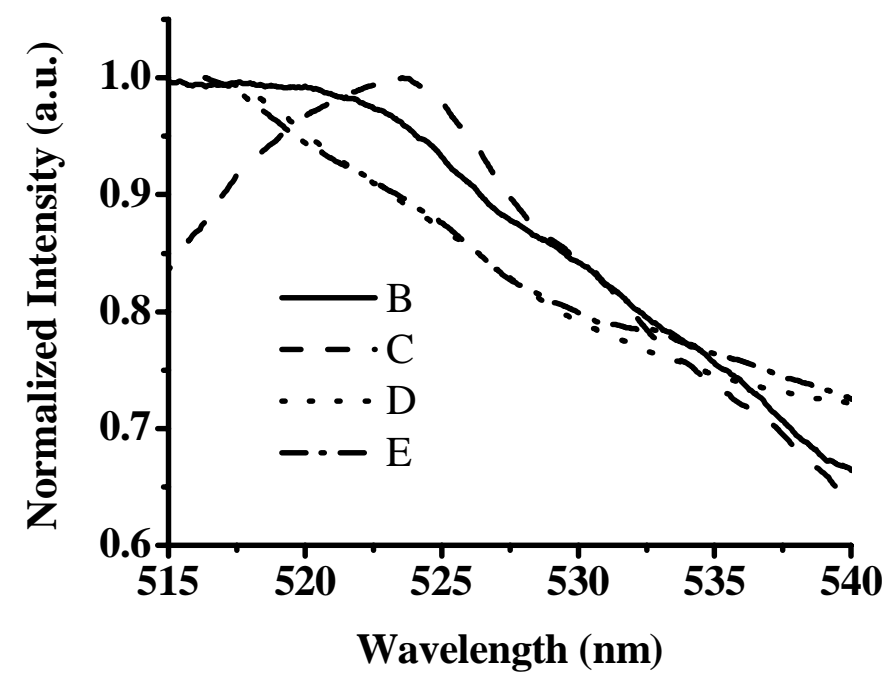

Fig. 7 Spectra taken at the beginning of decay from samples B, C, D and E. 


\section{CONCLUSIONS}

In summary, we have reported the cw and time-resolved PL results of the organic thin films that composes of the host emitting material $\mathrm{Alq}_{3}$ dopes with guest material C545T with different doping concentration. The dopant concentration has an optimized value at $2 \%$ with maximum PL efficiency. From time-resolved PL spectrum, at least two physical mechanisms were observed with different time constants involved in the quenching effect. The mechanism for the slow one was attributed to the nonradiative recombination, while that for the fast one might be due to the optical absorption of excimers or aggregates.

\section{ACKNOWLEDGMENTS}

This work was supported by the National Science Council, the Republic of China, under Grant No. NSC 92-2218-E-002-050.

\section{REFERENCES}

1. M. A. Baldo, M. E. Thompson, and S. R. Forrest, Nature (London) 403, 750 (2000).

2. X. Gong, M.R. Robinson, J.C. Ostrowski, D. Moses, G.C. Bazan, and A.J. Heeger, Adv. Mater. 14, 581 (2002).

3. X. Gong, J.C. Ostrowski, G.C. Bazan, D. Moses, and A.J. Heeger, Appl. Phys. Lett. 81, 3711 (2002).

4. R. A. Negres, X. Gong, J. C. Ostrowski, G. C. Bazan, D. Moses, and A. J. Heeger, Phys. Rev. B 68, 115209 (2003).

5. J. Kalinowski, W. Stampor, M. Cocchi, D. Virgili, V. Fattori, P. Di Marco, Chem. Phys. 297, 39 (2004).

6. R. N. Bera, Y. Sakakibara, M. Tokumoto and K. Yase, J. J. Appl. Phys. 42, 7379 (2003).

7. D. R. Lutz, K. A. Nelson, C. R. Gochanour and M. D. Fayer, Chem. Phys. 58, 325 (1981).

8. M. A. Baldo, and S. R. Forrest, Phys. Rev. B, 62, 10967 (2000).

9. D. Vacar, E. S. Maniloff, D. W. McBranch, and A. J. Heeger, Phys. Rev. B 56, 4573 (1997).

10. G. Cerullo, S. Stagira, M. Zavelani-Rossi, S.D. Silvestri, T. Virgili, D.G. Lidzey, and D.D.C. Bradley, Chem. Phys. Lett. 335, 27 (2001).

11. R. N. Bera, Y. Sakaibara, M. Tokumoto, and K. Yase, J. J. Appl. Phys. 42, 7379 (2003). 